\title{
NOTE ON A PROBLEM OF DICKSON
}

\author{
L. CARLITZ ${ }^{1}$
}

1. Let $q=p^{n}$, where $p$ is an odd prime. Let

$$
F(x)=a_{0} x^{k}+\cdots+a_{k} \quad\left(a_{j} \in G F(q), a_{0} \neq 0\right)
$$

be a polynomial of degree $k$ such that $F(\alpha)=\beta^{2}$, where $\beta \in G F(q)$ for all $\alpha \in G F(q)$. The writer $[1 ; 2]$ has proved the existence of a number $N_{k}$ such that if $q>N_{k}$ then

$$
F(x)=H^{2}(x) \quad(H(x) \in G F[q, x]) ;
$$

moreover $N^{k}$ satisfies

$$
N_{k} \leqq(k-1)^{2} .
$$

If $q=11$ and $F(x)=x^{5}+4$ it is easily verified that

$$
F(a) \equiv \begin{cases}5 \equiv 4^{2}(\bmod 11) & (a R 11) \\ 3 \equiv 5^{2}(\bmod 11) & (a N 11) .\end{cases}
$$

Clearly $F(x)$ is not congruent $(\bmod 11)$ to the square of a polynomial. We shall prove the following result.

TheOREM. The number $N_{k}$ satisfies

$$
N_{k}>2 k+1 \text {. }
$$

Proof. Put $q=2 m+1$ and consider the polynomial

$$
F(x)=x^{(q-1) / 2}+c \quad(c \in G F(q), c \neq 0) .
$$

Clearly $F(x)$ does not satisfy (1).

For $a \in G F(q)$ we define a real-valued function $\psi(a)$ by means of

$$
\psi(a)=\left\{\begin{aligned}
1 & \left(a^{m}=1\right) \\
-1 & \left(a^{m}=-1\right) \\
0 & (a=0)
\end{aligned}\right.
$$

To prove the theorem it will suffice to show the existence of a number $c \in G F(q)$ such that $\psi(F(a))=1$ for all $a \in G F(q)$. This is equivalent to the existence of $c$ such that

$$
\psi(c)=\psi(c+1)=\psi(c-1)=1 .
$$

Received by the editors November 13, 1961.

${ }^{1}$ Supported in part by National Science Foundation grant G 16485. 
Now when $q=p$ it is known $\left[3\right.$, p. 156] that the number $N_{0}(1,1,1)$ of incongruent $c(\bmod p)$ satisfying $(6)$ is determined by

$$
N_{0}(1,1,1)= \begin{cases}\frac{1}{8}(p-7) & (p \equiv-1(\bmod 8)) \\ \frac{1}{8}(p-3) & (p \equiv 3(\bmod 8))\end{cases}
$$

when $p \equiv 3(\bmod 4)$. When $p \equiv 1(\bmod 4)$ we have

$$
N_{0}(1,1,1)=\frac{1}{8}\left(p-3+\Phi_{p}\right)-1-\frac{1}{2}\left(\frac{2}{p}\right),
$$

where

$$
\Phi_{p}=\sum_{c=0}^{p-1}\left(\frac{c^{3}-c}{p}\right)
$$

moreover

$$
\left|\Phi_{p}\right| \leqq 2 p^{1 / 2}
$$

In the general case $\left(q=p^{n}\right)$ it is not difficult to show that

$$
N_{0}(1,1,1)= \begin{cases}\frac{1}{8}(q-7) & (q \equiv-1(\bmod 8)) \\ \frac{1}{8}(q-3) & (q \equiv 3(\bmod 8))\end{cases}
$$

when $q \equiv 3(\bmod 4)$. When $q \equiv 1(\bmod 4)$ we have

$$
N_{0}(1,1,1)=\frac{1}{8}\left(q-3+\Phi_{q}\right)-1-\frac{1}{2} \psi(2),
$$

where

$$
\Phi_{q}=\sum_{c \in G F(q)} \psi\left(c^{3}-c\right)
$$

and

$$
\begin{array}{rlrl}
\Phi_{q} & =0 & & (p \equiv 3(\bmod 4)) \\
\left|\Phi_{q}\right| \leqq 2 q^{1 / 2} & & (p \equiv 1(\bmod 4))
\end{array}
$$

It follows from (7) that

$$
N_{0}(1,1,1)>0
$$

for $q \equiv 3 .(\bmod 4), q>7$. For $p \equiv 3(\bmod 4)$ and $n$ even $(10)$ holds provided $q>15$. Finally when $p \equiv 1(\bmod 4),(10)$ holds provided

$$
\begin{aligned}
& q-15 \geqq 2 q^{1 / 2} \quad(q \equiv 1(\bmod 8)), \\
& q-7 \geqq 2 q^{1 / 2} \quad(q \equiv 5(\bmod 8)),
\end{aligned}
$$

that is, provided 


$$
\begin{array}{ll}
q \geqq 25 & (q \equiv 1(\bmod 8)), \\
q \geqq 13 & (q \equiv 5(\bmod 8)) .
\end{array}
$$

For the excluded small values of $q$ we take

$$
\begin{array}{ll}
F(x)=x^{8}+1 & (q=7), \\
F(x)=x^{4}+1 & (q=9), \\
F(x)=2 x^{6}+1 & (q=13), \\
F(x)=3 x^{8}+1 & (q=17) .
\end{array}
$$

Since a polynomial of the form

$$
F(x)=a x^{m}+b \quad(a, b \in G F(q), a b \neq 0)
$$

is clearly not equal to the square of a polynomial in $G F[q, x]$ the theorem follows.

Note that for $q>9$ we have proved the existence of a polynomial of the form (11) such that

$$
F(\alpha)=\beta^{2} \quad(\beta \in G F(q), \beta \neq 0)
$$

for all $\alpha \in G F(q)$.

2. In certain cases, at least, the lower bound (3) can be improved. For example if $q=4 m+1$ and we take

$$
F(x)=x^{m}+c \quad(c \in G F(q)),
$$

then for nonzero $a, a^{m}$ takes on one of the values $\pm 1, \pm \epsilon$, where $\epsilon^{2}=-1$. This leads to consideration of the sum

$$
\begin{aligned}
S=\sum_{c} & \{1+\psi(c)\}\{1+\psi(c+1)\}\{1+\psi(c-1)\} \\
\cdot & \{1+\psi(c+\epsilon)\}\{1+\psi(c-\epsilon)\},
\end{aligned}
$$

where the summation is over all $c \neq 1,-1, \epsilon,-\epsilon$. Using known estimates we find that $S>0$ provided $q$ exceeds a certain numerical bound (independent of $k$ ). It follows that

$$
N_{k}>4 k+1
$$

at least for $q \equiv 1(\bmod 4)$ and $k$ sufficiently large.

\section{REFERENCES}

1. L. Carlitz, A problem of Dickson's, Duke Math. J. 14 (1947), 1139-1140.

2. - A problem of Dickson, Duke Math. J. 19 (1952), 471-474.

3. H. Hasse, Vorlesungen uber Zahlentheorie, Springer-Verlag, Berlin, 1950.

DUKE UNIVERSITY 